\title{
In search of the nuclear clock
}

\section{A nuclear clock could outperform atomic clocks, but its development has turned out to be a formidable task, writes Marianna Safronova.}

$\mathrm{T}$ he pursuit of a nuclear clock has all the features of a quest: a grand idea, a unique treasure, a number of false sightings, a controversy, a long period of (seemingly) fruitless searching and, finally, a pathway toward the ultimate goal.

What is a nuclear clock? The basic principle behind any type of clock is a periodic event, defining one clock 'tick'. In addition, one needs a device that can be tuned to the periodic signal, as well as a procedure for counting the clock ticks. Our present definition of a second is based on a perfect, naturally occurring oscillator: an electronic transition between particular energy levels of a caesium atom. Electronic transitions in other atoms, with much higher frequencies $\left(4 \times 10^{14}-1.3 \times 10^{15} \mathrm{~Hz}\right)$ than the caesium transition $\left(9 \times 10^{9} \mathrm{~Hz}\right)$, lying in the optical range and excited by an ultrastable laser, now form the basis of even more precise atomic clocks, which will neither lose nor gain 1 second in 15 billion years ${ }^{1}$. The nuclear clock would be based on a transition between the energy levels of a nucleus rather than electronic energy levels. A great benefit of this concept is its reduced sensitivity to the external perturbations that cause systematic errors in present atomic clocks, such as electric and magnetic fields, electric field gradients and blackbody radiation - these could be avoided or at least suppressed in a nuclear clock ${ }^{2,3}$.

Alas, nuclear transition frequencies are typically many orders of magnitude higher than those accessible by lasers! But here comes our treasure - a unique exception: a long-lived nuclear transition that occurs between an excited state (isomer) of the ${ }^{229} \mathrm{Th}$ isotope and the corresponding nuclear ground state (pictured). Its frequency is expected to be between 150 and $170 \mathrm{~nm}$, within reach of modern lasers. This transition eluded scientists during years of efforts to achieve its laser excitation; partly due to the large uncertainty in the transition frequency and its long lifetime, which is expected to be about $10^{4}$ seconds. Even the very existence of the ${ }^{229 \mathrm{~m}} \mathrm{Th}$ isomer

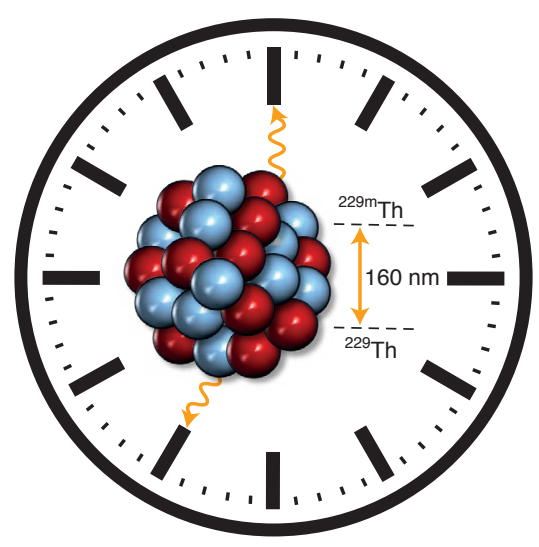

came into question after unconfirmed and disputed sightings ${ }^{2}$, but it was finally definitively confirmed ${ }^{4}$ in 2016 . Experiments to verify and pinpoint the wavelength of the transition from the isomer to the nuclear ground state are ongoing ${ }^{2}$. The hyperfine structure (splitting of the atomic energy levels due to the interaction of electrons with nuclear electric and magnetic moments) of the isomer was recently observed in another breakthrough experiment ${ }^{5}$ also providing further insights into the isomer's fundamental nuclear properties.

These recent experimental milestones ${ }^{4,5}$ have provided a solid base for progress toward the realization of an actual nuclear clock. Two different types of nuclear clock design have been proposed, one based on trapped ions and another on solid-state systems ${ }^{2}$. The former involves a single trapped Th atom, with one or more electrons removed, and technologies similar to those used in current trapped-ion clocks ${ }^{1}$. This scheme offers unprecedented suppression of systematic shifts of the measured clock frequency, and an estimated clock performance with a total fractional inaccuracy of $1 \times 10^{-19}$ (ref. 3).

In the other, completely different proposal, thorium is implanted in crystals transparent to radiation in the vacuum-ultraviolet range of the ${ }^{229} \mathrm{Th}$ nuclear clock transition, such as calcium fluoride. An advantage of such a scheme is the possibility to have a large number of Th nuclei (well beyond $10^{10}$ ) in a solid, resulting in a significant gain in signalto-noise ratio and clock stability ${ }^{2}$.

The development of ever more precise and stable clocks is of great importance for many purposes ${ }^{1}$, ranging from practical applications in geodesy to testing cornerstones of modern physics, such as the constancy of fundamental constants and Lorentz invariance. The variation of fundamental constants is an element of many theories beyond the standard model of particle physics and might be caused by the so-called dark matter ${ }^{6}$ - the 'missing' matter in the Universe. It has been suggested ${ }^{7}$ that the clock transition in the ${ }^{229} \mathrm{Th}$ nucleus is enormously (by five orders of magnitude) more sensitive to the variation of the fine-structure constant and quark masses than all atomic clocks currently in operation. This result is presently a matter of controversy, with widely disparate theoretical estimates. A way to resolve this problem and to determine the sensitivity has recently been proposed, involving measurements of the nuclear quadrupole moment and the nuclear charge radius of the ${ }^{229 \mathrm{~m}} \mathrm{Th}_{\mathrm{isomer}}$. First experimental results have been just reported ${ }^{5}$.

The quest for the nuclear clock continues and the next decade may see the exploitation of a nuclear transition for new levels of metrological accuracy as well as insights into our Universe.

MARIANNA SAFRONOVA is in the Department of Physics and Astronomy, University of Delaware, Newark, Delaware 19716, USA.

e-mail:msafrono@udel.edu

\section{References}

1. Ludlow, A. D., Boyd, M. M., Ye, J., Peik, E. \& Schmidt, P. O. Rev. Mod. Phys. 87, 637-701 (2015).

2. Peik, E. \& Okhapkin, M. C. R. Phys. 16, 516-523 (2015).

3. Campbell, C. J. et al. Phys. Rev. Lett. 108, 120802 (2012).

4. von der Wense, L. et al. Nature 533, 47-51 (2016).

5. Thielking, J. et al. Preprint at https://arxiv.org/abs/1709.05325 (2017).

6. Arvanitaki, A., Huang, J. \& Van Tilburg, K. Phys. Rev. D 91, 015015 (2015).

7. Berengut, J. C., Dzuba, V. A., Flambaum, V. V. \& Porsev, S. G. Phys. Rev. Lett. 102, 210801 (2009).

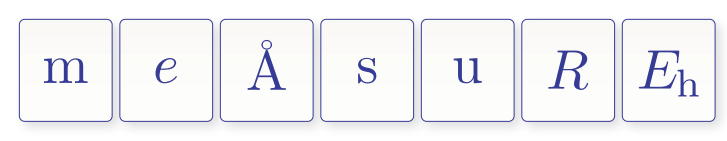

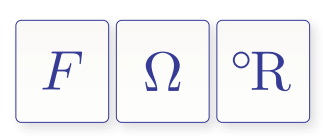

$\varepsilon_{0}$

$\alpha$

$\sigma$

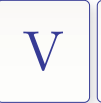

$\mathrm{R}$ 